\title{
Hot-spot detection and calibration of a scanning thermal probe with a noise thermometry gold wire sample
}

\author{
Angelo Gaitas, ${ }^{1,2, a)}$ Steven Wolgast, ${ }^{3}$ Elizabeth Covington, ${ }^{3}$ and Cagliyan Kurdak $^{3}$ \\ ${ }^{1}$ PicoCal, Inc., 333 Parkland Plaza, Ann Arbor, Michigan 48103, USA \\ ${ }^{2}$ Department of Microelectronics and Computer Engineering, Delft University of Technology, Mekelweg 4, \\ Delft, The Netherlands \\ ${ }^{3}$ Physics Department, University of Michigan, 450 Church Street, Ann Arbor, Michigan 48109, USA
}

(Received 27 August 2012; accepted 4 February 2013; published online 20 February 2013)

\begin{abstract}
Measuring the temperature profile of a nanoscale sample using scanning thermal microscopy is challenging due to a scanning probe's non-uniform heating. In order to address this challenge, we have developed a calibration sample consisting of a 1- $\mu \mathrm{m}$ wide gold wire, which can be heated electrically by a small bias current. The Joule heating in the calibration sample wire is characterized using noise thermometry. A thermal probe was scanned in contact over the gold wire and measured temperature changes as small as $0.4 \mathrm{~K}$, corresponding to $17 \mathrm{ppm}$ changes in probe resistance. The non-uniformity of the probe's temperature profile during a typical scan necessitated the introduction of a temperature conversion factor, $\eta$, which is defined as the ratio of the average temperature change of the probe with respect to the temperature change of the substrate. The conversion factor was calculated to be $0.035 \pm 0.007$. Finite element analysis simulations indicate a strong correlation between thermal probe sensitivity and probe tip curvature, suggesting that the sensitivity of the thermal probe can be improved by increasing the probe tip curvature, though at the expense of the spatial resolution provided by sharper tips. Simulations also indicate that a bow-tie metallization design could yield an additional 5- to 7-fold increase in sensitivity. (C) 2013 American Institute of Physics. [http://dx.doi.org/10.1063/1.4792656]
\end{abstract}

\section{INTRODUCTION}

Thermal measurements at the nanometer scale are of both scientific and industrial interest, and over the past two decades, scanning thermal microscopy has been used in a variety of applications. ${ }^{1-10}$ Temperature calibration is required for scanning thermal microscopy, where substrate temperature changes must be measured with high accuracy. A number of ways to calibrate a scanning thermal probe have been developed ${ }^{11,12}$ and include: isothermal (hotplate) calibration, ${ }^{13}$ melting point standard calibration, ${ }^{14,15}$ use of the linearity of heater resistance with temperature, ${ }^{16}$ calibration methodologies using Raman thermometry, ${ }^{11}$ and the use of a small thermocouple in contact with the probe. ${ }^{18}$ Many calibration techniques measure the temperature changes of the probe while operating the probe as a heater; however, this does not account for the heat transfer effects at the substrate-tip interface during a thermal scan.

In macroscopic thermometry, the thermometer is typically smaller than the sample measured, allowing measurements with high accuracy. In contrast, a scanning probe is much larger than the microscopic dimensions of the measured area of the sample. Thus, the probe is non-uniformly heated, and the average temperature change of the probe is only a small fraction of the sample's actual temperature variation. To properly characterize the response of the probe, it can be scanned along a calibration sample with a known temperature profile.

In this paper, a pre-calibrated $1-\mu \mathrm{m}$-wide gold wire sample on a silicon-oxide substrate is used as a Joule heater to directly calibrate a scanning thermal probe. This calibration wire is a Johnson noise thermometer, i.e., a primary

a)Tel.: 734-913-2608. Electronic mail: angelo@picocal.com. thermometer; therefore, the wire does not require calibration by another external device. The calibration wire is heated by passing current through it. At each temperature, the probe is scanned over the wire. The change in resistance of the probe with temperature is used as a calibration of the thermal probe, which is quantified by introducing a temperature conversion factor, $\eta$.

This technique offers several advantages. For example, the scanning thermal probe can be calibrated directly from temperature changes on the sample, thereby obtaining a direct correlation of resistance changes of the probe with temperature changes of the sample. The probe and the calibration wire do not get contaminated, and they can be re-used. The calibration wire has a very long shelf life because it is made of gold, which does not oxidize. In addition, it is good practice to calibrate each probe individually before use, because scanning thermal probes are not identical due to tip-curvature and film-thickness variations arising during the microfabrication process.

Finally, finite element analysis (FEA) simulations indicate a strong correlation between thermal probe sensitivity and probe tip curvature. The simulations and experimental results suggest that the thermal sensitivity of the probes can be improved by increasing the probe tip curvature, using materials other than gold as sensing elements, and by changing the geometry of the sensing element over the tip. Specifically, we investigated a bow-tie metallization design that increases the sensitivity 5- to 7-fold in simulations.

\section{SCANNING THERMAL PROBE}

For this study, we used a micro-machined scanning thermal probe ${ }^{17-19}$ that includes two embedded sensors-one for 
measuring deflection, and another for measuring localized heating. In a typical application, the temperature profile of the sample is obtained by monitoring the sensor resistance during a probe scan. The scanning thermal probe is micromachined in a four-mask fabrication process. ${ }^{17-19}$ The cantilever is a stacked structure of $\mathrm{Si}$ and $\mathrm{SiO}_{2}$ layers $300 \mu \mathrm{m}$ long, $200 \mu \mathrm{m}$ wide, and $2 \mu \mathrm{m}$ thick, as shown in the scanning electron microscopy (SEM) image Figure 1(a). The two sensing elements are 10-nm-thick gold films. The elastoresistive element is $20 \mu \mathrm{m}$ wide (situated near the base of the cantilever), and the thermal element is $30 \mu \mathrm{m}$ wide, which narrows to $10 \mu \mathrm{m}$ over the tip. Using a thin and narrow metal as a sensing element forces the detection current through a narrow area on the surface of the tip, increasing temperature sensitivity by localizing the measurement to the surface of tip. The tip (Figure 1(b)) is $6-7 \mu \mathrm{m}$ high and has a radius of curvature that ranges between $50 \mathrm{~nm}$ and $500 \mathrm{~nm}$. A $3 \times 1.4 \times 0.5-\mathrm{mm}^{3}$ chip serves as the base of the cantilever.

The temperature coefficient of resistance (TCR) of the scanning thermal probe is given by

$$
\alpha_{p}=\frac{R_{P}(T)-R_{P}\left(T_{0}\right)}{R_{P}\left(T_{0}\right)\left(T-T_{0}\right)},
$$

where $\alpha_{p}$ is the TCR of the probe at temperature $T_{0}$, and $R_{P}(T)$ is the resistance of the probe at temperature $T$. The TCR of these probes is approximately $0.001 \mathrm{~K}^{-1}$. The TCR was measured by enclosing the probe and a calibrated thermistor in an aluminum chamber that rested on a hot plate, which allowed the probe temperature to be varied uniformly while the temperature and resistance of the probe were recorded simultaneously. Because the sensing element is made of gold, the TCR of the probe is not very high; however,

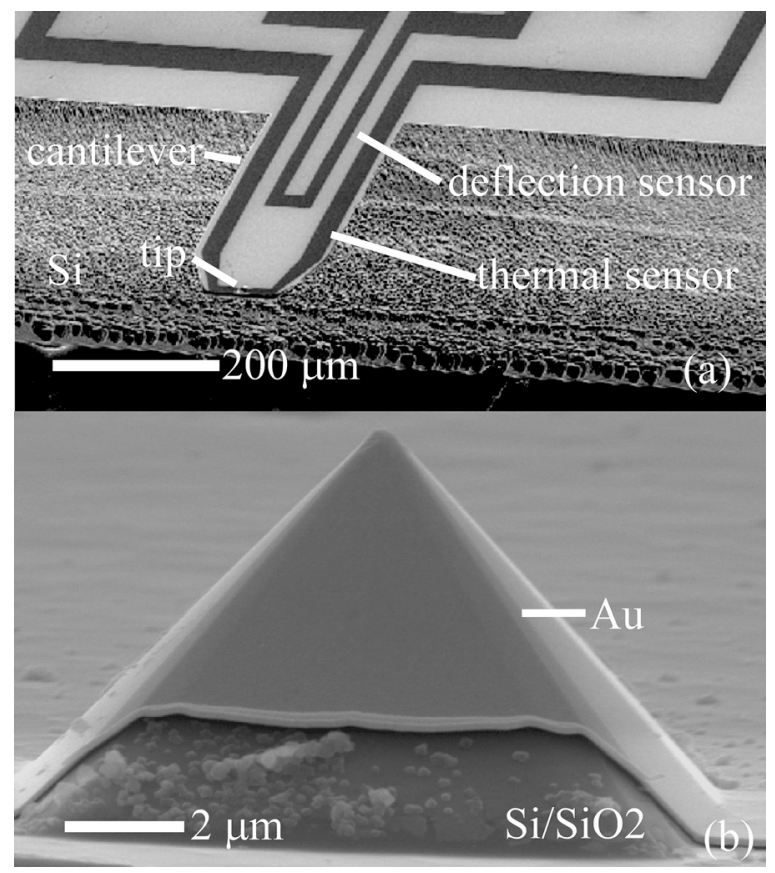

FIG. 1. (a) SEM image of the thermal scanning probe. (b) SEM image of the active region of the tip. The Au layer does not cover the entire tip, but is mostly concentrated in the active area. The tip has a radius of curvature between $50 \mathrm{~nm}$ and $500 \mathrm{~nm}$. gold is advantageous because it does not oxidize, and therefore has a long shelf life.

The TCR calibration alone is not sufficient to determine the real temperature change of the sample because the temperature of the probe is highly non-uniform. Additionally, each probe must be calibrated individually, since the curvature of the tip can influence the measurement. Therefore, there is a need for a calibration sample to simplify individual probe calibration.

\section{GOLD WIRE CALIBRATION}

\section{A. Device and fabrication}

A four-terminal linear gold resistor is used as a calibration device to distinguish the thermal noise generated in the lead wires from that generated in the resistor. SEM images of the wires are shown in Figure 2. The device rests on 300$\mathrm{nm} \mathrm{SiO}_{2}$ grown on a $500-\mu$ m-thick $\mathrm{Si}$ wafer. The sample pattern includes two parallel wires that are $400 \mu \mathrm{m}$ long, $1 \mu \mathrm{m}$ wide, and $15 \mathrm{~nm}$ thick. The thickness was chosen to produce an adequate nominal resistance for noise thermometry and to offer a low profile so that the thickness of the metal does not affect the thermal measurement. There is a $1-\mu \mathrm{m}$ gap between the two wires. Each wire is connected to four leads and contact pads: one on each end of the wire, and one positioned $50 \mu \mathrm{m}$ from each end. The wire had a resistance of $993 \Omega$.

\section{B. Noise thermometry}

The noise power spectral density of a resistor in thermal equilibrium at a temperature $T$ is given by

$$
S_{V}^{\text {Johnson }}(f)=4 k_{B} T R
$$

where $k_{B}$ is Boltzmann's constant and $R$ is the resistance of the device. Since the thermal noise is directly proportional to temperature, it is an attractive means of measuring temperature. A noise thermometer is only dependent on the resistance of the sensor, which is easily measured. It is a primary thermometer, i.e., it does not need to be calibrated using other thermometers or standard references.

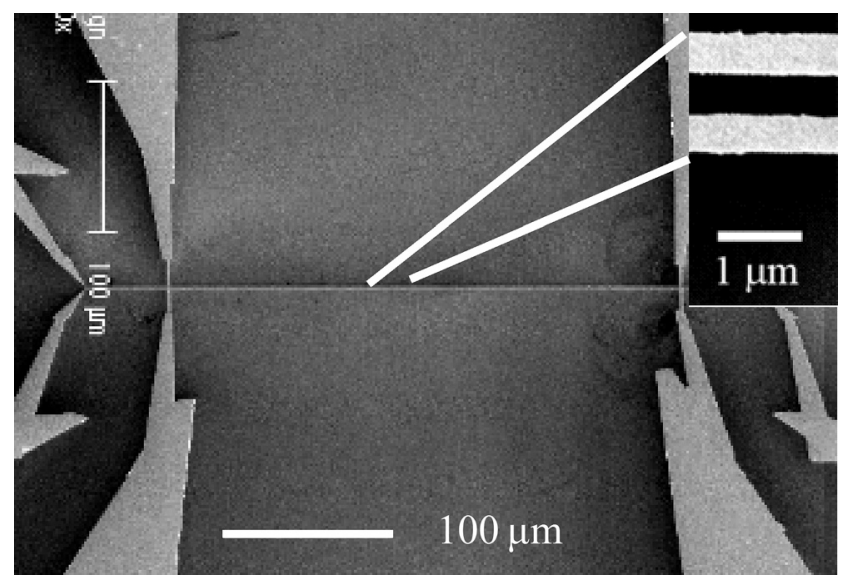

FIG. 2. SEM image of the calibration sample, which consists of 2 parallel gold wires that are $400 \mu \mathrm{m}$ long, $1 \mu \mathrm{m}$ wide, and $15 \mathrm{~nm}$ thick. 
In this work, $S_{V}(f)$ of the gold wire sample is measured using a low-noise preamplifier (Stanford Research Systems, model SR560) and a spectrum analyzer (Stanford Research Systems, model SR780).

The circuit in Figure 3 was used to measure the noise power spectrum of the gold wire sample. A low-noise battery was used to pass a current via the outer contact pads. The inner contact pads were used to measure the noise power spectral density function with a spectrum analyzer. The bias resistor was $3 \mathrm{k} \Omega$.

We consider three sources of noise: amplifier noise, Johnson noise, and $1 / \mathrm{f}$ noise. The noise referred to the input of the amplifier is, thus, given by

$$
S_{V}(f)=S_{V}^{A m p}(f)+S_{V}^{J \text { ohnson }}(f)+S_{V}^{1 / f}(f),
$$

where

$$
S_{V}^{1 / f}(f)=\frac{A I^{2}}{f}
$$

where $I$ is the current through the resistor and gold wire sample, and $A$ is a constant. The Johnson noise for the resistor and the gold wire sample each is given by Eq. (2).

The DC bias has very low noise, so it is neglected in the AC circuit analysis. To calculate the Johnson noise referred to the input of the amplifier, first the AC input voltage is calculated by

$$
V_{\text {input }}=V_{B}\left(1-\frac{R_{B}}{R_{H}+R_{B}}\right)-V_{H} \frac{R_{B}}{R_{H}+R_{B}},
$$

where $V_{B}$ and $V_{H}$ are the voltage noise of the bias resistor and gold wire sample, respectively, and $R_{B}$ and $R_{H}$ are the resistances of the bias resistor and gold wire sample, respectively. Then, the following is obtained:

$$
S_{V}^{\text {Johnson }}=S_{V B}\left(1-\frac{R_{B}}{R_{H}+R_{B}}\right)^{2}-S_{V H}\left(\frac{R_{B}}{R_{H}+R_{B}}\right)^{2},
$$

where $S_{V B}$ and $S_{V H}$ are the individual values of the Johnson noise from the bias resistor and gold wire sample, respectively. Thus,

$$
\begin{aligned}
S_{V}^{\text {Johnson }}= & 4 k_{B} T_{0} R_{B}\left(1-\frac{R_{B}}{R_{H}+R_{B}}\right)^{2} \\
& +4 k_{B} T_{H} R_{H}\left(\frac{R_{B}}{R_{H}+R_{B}}\right)^{2},
\end{aligned}
$$

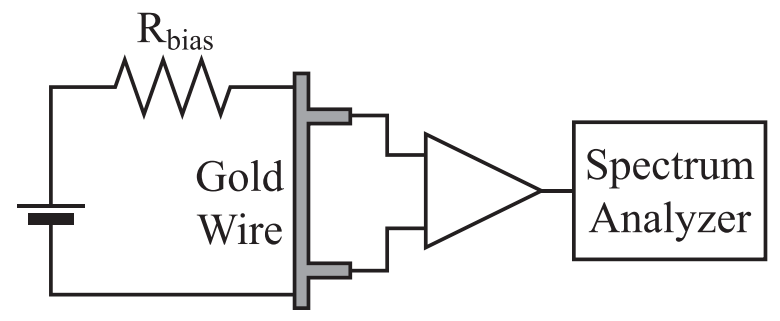

FIG. 3. Schematic of the experimental setup for noise thermometry measurements. where $T_{0}$ is the absolute ambient temperature, and $T_{H}=T_{0}$ $+\Delta T$, where $\Delta T$ is the excess temperature of the gold wire sample above the ambient temperature. It should be noted that $R_{H}$ is dependent on temperature linearly to a good approximation over a short temperature range, and is expressed as

$$
R_{H}=\left(1+\alpha_{w} \Delta T\right) R_{H 0},
$$

where $\alpha_{w}$ is the experimentally determined TCR of the gold wire, and $R_{H 0}$ is $R_{H}$ measured at ambient temperature. The TCR $\alpha_{w}$ is $1711 \mathrm{ppm} / \mathrm{K}$. For the temperature range of interest, the contribution from the TCR is negligible in the denominators of the expression for Johnson noise. Thus, to a good approximation, $S_{V}^{\text {Johnson }}$ can be rewritten as

$$
S_{V}^{J o h n s o n}=4 k_{B} T_{0} R_{e q}+4 k_{B} \Delta T \frac{R_{e q}^{2}}{R_{H 0}}\left(1+\alpha_{w} T_{0}+\alpha_{w} \Delta T\right),
$$

where $R_{e q}$ is the Thevenin equivalent resistance of the circuit at the input of the amplifier and is independent of temperature. The total noise referred to the input of the amplifier is then given by

$$
\begin{aligned}
S_{V}(f)= & S_{V}^{A m p}(f)+4 k_{B} T_{0} R_{e q} \\
& +4 k_{B} \Delta T \frac{R_{e q}{ }^{2}}{R_{H 0}}\left(1+\alpha_{w} T_{0}+\alpha_{w} \Delta T\right)+\frac{A I^{2}}{f} .
\end{aligned}
$$

The first two terms are independent of current. Thus, by measuring the total noise as a function of current, then subtracting the $1 / \mathrm{f}$ term, $\Delta T$ may be determined. The second two terms are both zero when $I=0$, so the excess noise can be defined as

$$
\begin{aligned}
S_{V}^{E x c e s s}(f, I) & \equiv S_{v}(f, I)-S_{v}(f, I=0)-\frac{A I^{2}}{f} \\
& =4 k_{B} \Delta T \frac{R_{e q}{ }^{2}}{R_{H 0}}\left(1+\alpha_{w} T_{0}+\alpha_{w} \Delta T\right) .
\end{aligned}
$$

The average excess noise power density can be plotted against $I^{2}$ (Figure 4) to obtain the dependence of $\Delta T$ as a function of $I^{2}$. The maximum DC current applied (4.5 mA) corresponds to an excess temperature of $16.3 \mathrm{~K}$. Since the excess temperature scales quadratically with the applied current, the temperature change corresponding to other values of the applied current can be calculated using the following equation:

$$
T=\frac{T_{\max } I^{2}}{I_{\max }^{2}} .
$$

\section{EXPERIMENTAL RESULTS AND DISCUSSION}

\section{A. Thermal probe calibration and hot-spot scans}

A scanning system was developed for these measurements. The system includes a closed-loop piezoelectric XYZ 


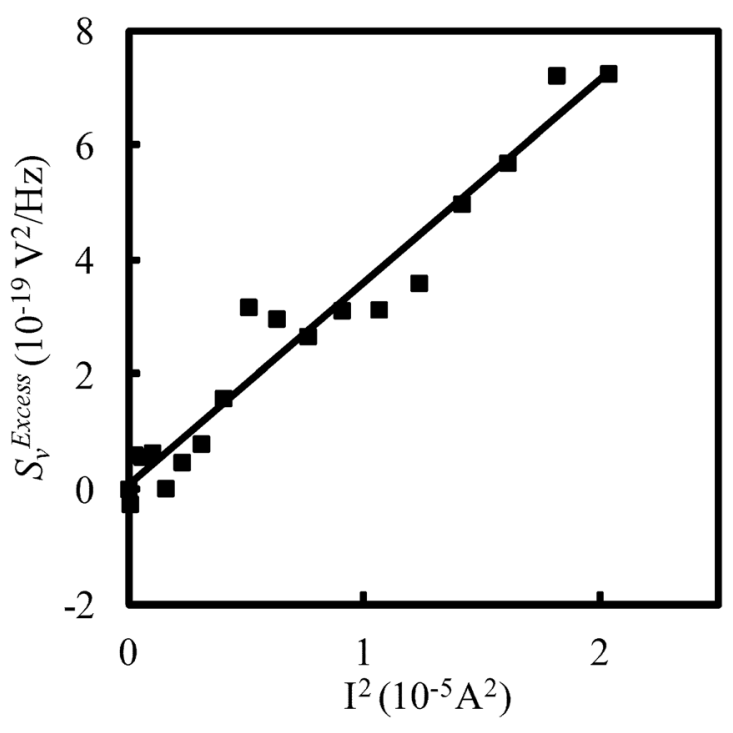

FIG. 4. Average excess white noise power density vs. $\mathrm{I}^{2}$. The experimental data (points) are linear, and a fit (line) gives a slope of $3.53^{-14} \mathrm{~V}^{2} / \mathrm{A}^{2} \mathrm{~Hz}^{1 / 2}$. At $4.5 \mathrm{~mA}$, the increase in temperature is $16.3 \mathrm{~K}$.

stage with a $100-\mu \mathrm{m}$ range on each axis and nanometer resolution (PiezoJena, Tritor 100), and an XYZ motorized stage with a motion range of $28 \mathrm{~mm}$ on each axis and $0.05-\mu \mathrm{m}$ microstep resolution (Zaber, model KT-LS28-MV). The system included a holder for the scanning thermal probe. The sample was brought into contact with the probe as the probe was monitored optically via a microscope and electrically via the response of the deflection-sensing element. The resistive change of the sensing element was directly measured using a micro-Ohm meter (Agilent, model $34420 \mathrm{~A}$ ) without a pre-amplifying circuit. The data were acquired with a LABVIEW program.

Figure 5(a) shows a two-dimensional XY plot of the scanning thermal probe's resistance over the wire with $\Delta T=1.6 \mathrm{~K}$. It is important to note that the thermal image is not polluted by the topography since there is an identical unheated gold wire next to the gold wire that is not observed in the thermal scan. The minimum detectable change in temperature of the sample is $0.4 \mathrm{~K}$, corresponding to a $17 \mathrm{ppm}$ change in resistance of the probe (the probe has a nominal resistance of $542.6 \Omega$ ). There is a small but detectable upward drift in the resistance of the probe, which is due to environmental temperature fluctuations during the scans. Figure 5(b) shows the measured peak $\Delta R$ as a function of applied bias currents squared, demonstrating that the probe signal is due to the excess temperature of the gold wire. A typical metal sensing element exhibits a linear behavior with temperature, accordingly temperature differentials of up to $2.5 \mathrm{~K}$ were used. There might be more complexities at higher temperatures due to changes in heat transfer mechanisms. Cantilever deformation effects were not observed due to the wire's small temperature differentials and the small current values through the probe. The line scan inset in Figure 5(b) indicates an increase in probe resistance of $0.03 \Omega$, corresponding to a wire temperature change of $1.6 \mathrm{~K}$, when the probe is scanned perpendicular to the wire.

The temperature distribution of the probe is nonuniform because the hot-spot area of the tip is much smaller
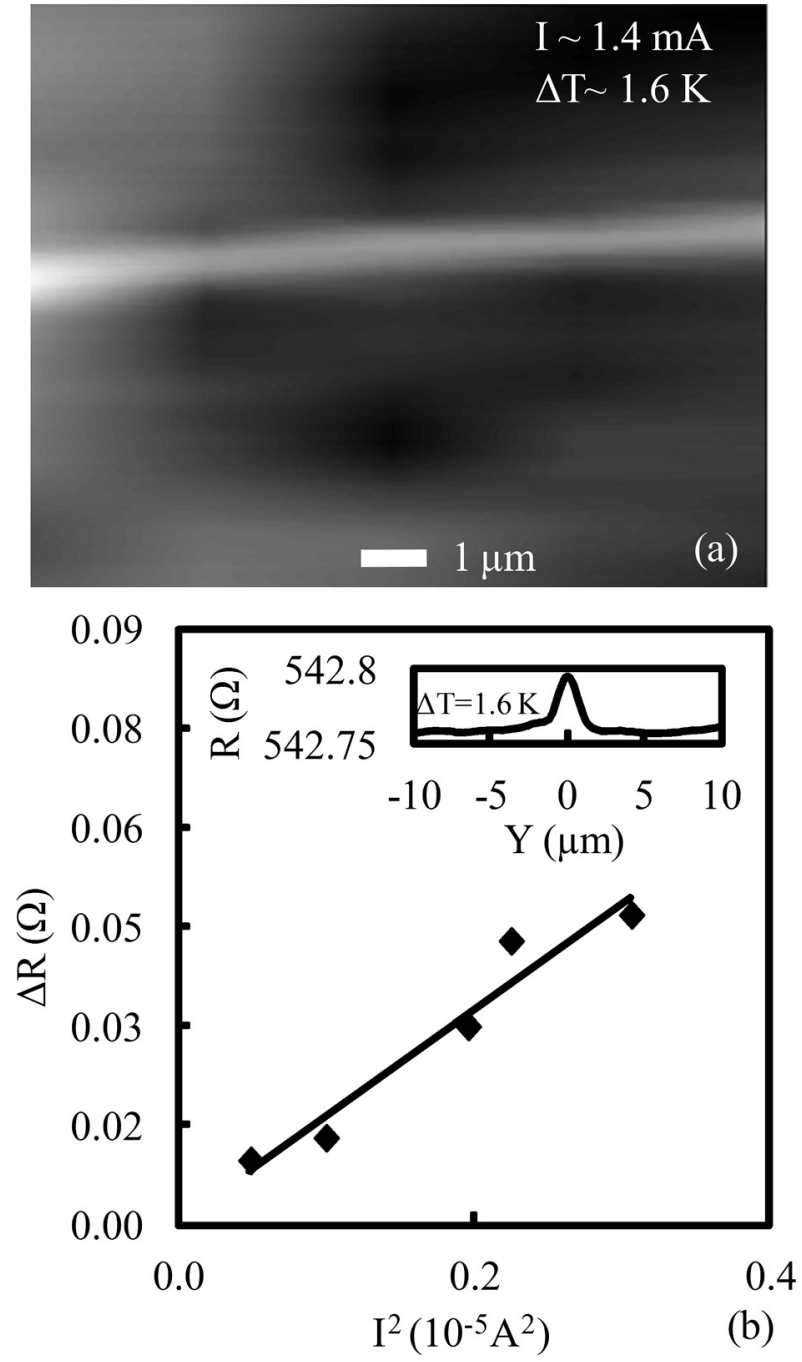

FIG. 5. (a) A two-dimensional XY plot of the scanning thermal probe's resistance change over the wire with $\Delta T$ of $1.6 \mathrm{~K}$. (b) The plot of the measured peak height, $\Delta R$, as a function of bias currents squared applied at the wire shows that the probe signal is due to the excess temperature of the wire. Inset: Line scan of the probe over the gold wire at $\Delta T_{\text {wire }}=1.6 \mathrm{~K}$, corresponding to $\Delta R_{\text {probe }}=0.03 \Omega$. The probe is scanned perpendicular to the wire.

than the entire probe tip area. As a result, the average temperature change of the probe is much lower than the temperature variation of the hot-spot on the substrate. This motivated the introduction of a temperature conversion factor $\eta$ such that

$$
\Delta T_{\text {probe }}=\eta \Delta T_{\text {wire }}
$$

where $\Delta T_{\text {probe }}$ is the average change of the probe's temperature. Experimentally, $\eta$ can be determined from the TCR of the scanning thermal probe, $\Delta R_{\text {probe }}$, and $\Delta T_{\text {sample }}$ (which are obtained during the calibration scan). $\eta$ can then be used in future scans to calculate the real temperature change of a sample from the measured changes in $\Delta R_{\text {probe }}$. Using Eqs. (1) and (13), the average value of the temperature conversion factor $\eta$ is $0.035 \pm 0.007$. A FEA model based on heat transfer theory was developed to determine what influences the value of $\eta$ and how it can be improved. 


\section{B. FEA simulations}

The mechanisms of heat transfer from the substrate to the tip ${ }^{20,21}$ are: solid-solid conduction, solid-gas conduction through the air gap, convection through the air gap, radiation from the heated substrate, and conduction through the liquid meniscus that forms between the tip and the substrate. Of these, solid-solid conduction is the dominant mode of heat transfer. The effects of sample heating on the probe's sensing element were simulated with a 2D comsol MULTIPHYSICs FEA model. These results were then used to derive a theoretical value for $\eta$. This model neglects heat transfer by radiation.

In the model, the $7-\mu \mathrm{m}$ tip is composed of a $10-\mathrm{nm}$ gold surface layer, a $100-\mathrm{nm} \mathrm{SiO}_{2}$ intermediate layer, and the silicon inner layer. The top of the tip is set at room temperature $(293 \mathrm{~K})$. The tip rests on a $1-\mu \mathrm{m}$-wide and 25 -nm-thick gold wire set at $294 \mathrm{~K}$. The gold wire rests on a $600-\mu \mathrm{m}$-wide, 300 -nm-thick $\mathrm{SiO}_{2}$ insulating layer on 300- $\mu$ m-thick silicon. A 240-nm-wide liquid meniscus surrounds the probe tip at the contact point. The probe and gold wire are surrounded by air, which is set to have an open boundary and is convectively cooled. The wall boundaries of surfaces of the solid materials that are exposed to fluids (water and air) account for convection. Seven cases with and without the presence of the liquid meniscus were considered, including tips with radii of curvature ranging from $100 \mathrm{~nm}$ to $700 \mathrm{~nm}$.

A close-up of the temperature profile of the metal sensing element on a tip with a $300-\mathrm{nm}$ radius of curvature is shown in Figure 6 . The contact area is $<10 \mathrm{~nm}$. The hot-spot size of the probe extends a few nanometers around the point of contact. However, the temperature of the entire probe is only slightly elevated above the ambient temperature. As the probe tip radius increases, the probe sensitivity to substrate temperature also increases.

Plots of the surface temperature of metal element of tip with varying radii vs. distance $\mathrm{X}$ along the horizontal axis from the contact point are shown in Figure 7. The simulations indicate that the temperature distribution is highly non-

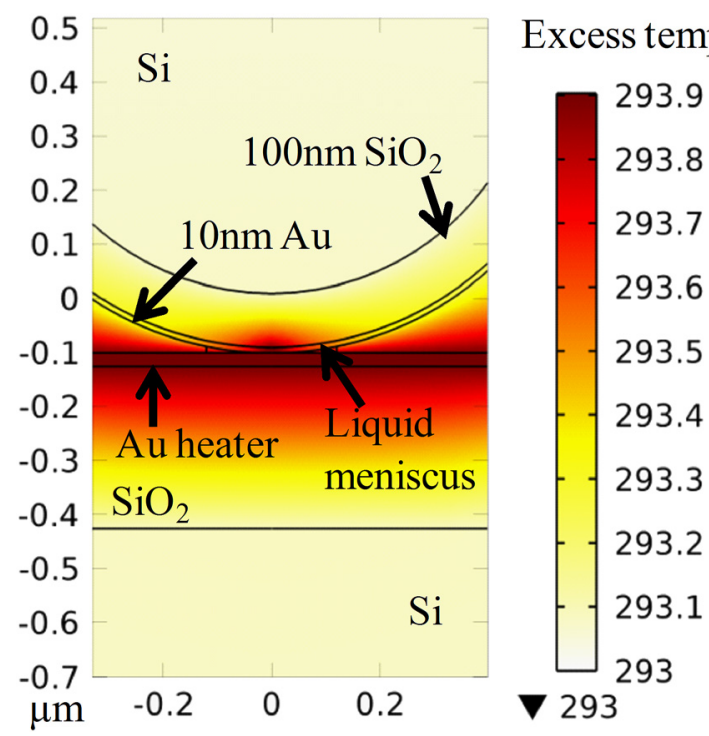

FIG. 6. Cross-sectional temperature distribution of a 600-nm radius tip.

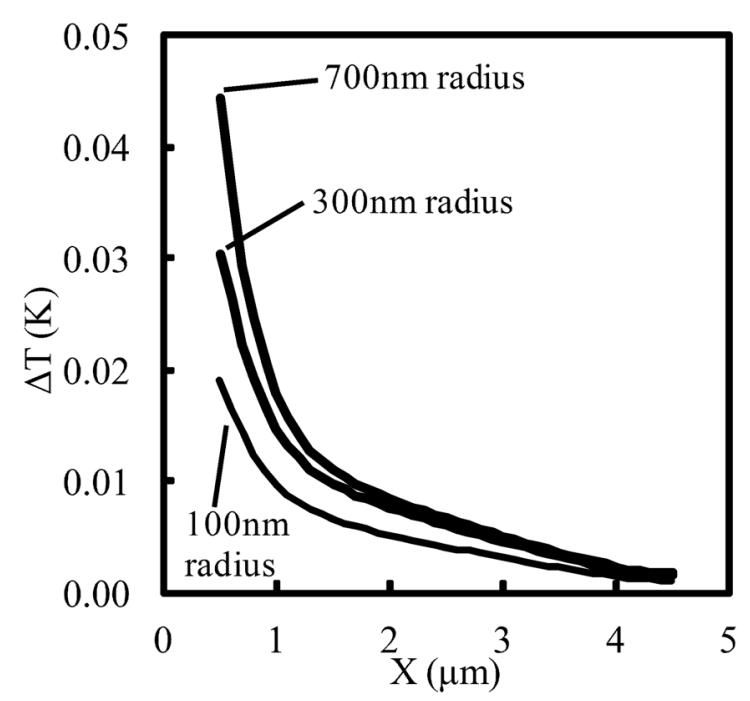

FIG. 7. A plot of the surface temperature of the metal element versus distance $\mathrm{X}$ along the horizontal axis from the contact point for the three values of tip radii: $100 \mathrm{~nm}, 300 \mathrm{~nm}$, and $700 \mathrm{~nm}$.

uniform and depends on the tip's curvature. The tip with 700-nm radius exhibits the highest average temperature among the curvatures studied. The temperature of the sensing element sharply drops to room temperature at a distance of about $1 \mu \mathrm{m}$ from the contact point. The hot-spot area is very small compared to the entire probe tip area.

The spatially averaged change in the temperature of the 10-nm gold sensing element can be determined from the simulation results. In this calculation, we assume that the measured resistance is dominated by the tip region. The lead and contact resistances are not included in the calculations. The following equation is used, assuming a conical tip, to calculate the spatially averaged temperature:

$$
\langle T\rangle=\frac{1}{\pi r_{0}^{2}} \int_{0}^{r_{0}} 2 \pi r T(r) d r .
$$

The temperature conversion factor, $\eta$, is then calculated and ranges from 0.006 to 0.012 , increasing with the radius of curvature of the tip (Figure 8). These results indicate that there is a compromise between spatial resolution and temperature sensitivity in the probe design. Sharper probe tips permit higher spatial resolution but lower temperature sensitivity.

The experimentally determined value of $\eta$ is larger than the values obtained from the FEA model. This discrepancy could be explained by a larger contact area between the tip and the sample, a larger liquid meniscus around the tip, or a larger probe tip radius.

Using FEA, we studied alternative probe geometries that yield higher values of $\eta$. One simple way to enhance probe performance is to use a bow-tie metallization design, shown in Figure 8, which focuses the current to the area in contact with the sample. Compared to the previous design, the bow-tie design we simulated produces a 5- to 7-fold increase in $\eta$. Sensitivity can be further increased by depositing a thinner metal film over the tip, which often enhances the film's TCR, and using different metals with higher TCR's. 


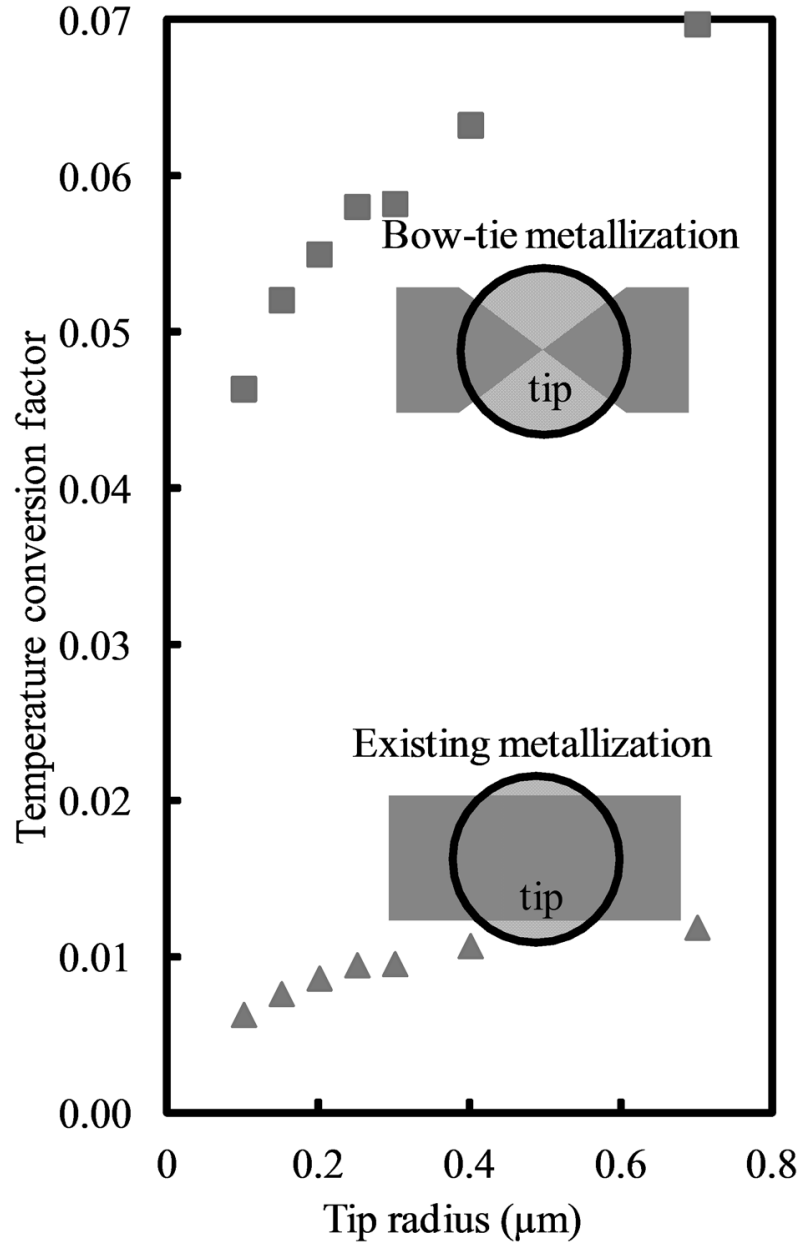

FIG. 8. Temperature conversion factor, $\eta$, versus tip radius for two different thermal probe patterns.

\section{CONCLUSIONS}

In this paper, we have addressed a key challenge of temperature profiling by developing a technique to accurately calibrate scanning thermal probes using a $1-\mu \mathrm{m}$-wide gold wire. The gold wire was pre-calibrated using noise thermometry. It was then used to obtain a direct correlation of resistance changes of the probe with temperature changes of the wire. This technique allows users to account for the non-uniform heating of the probe. Sample temperature changes as small as $0.4 \mathrm{~K}$ were detected, corresponding to 17 -ppm changes in probe resistance.

A temperature conversion factor, $\eta$, was introduced to help us compare the average change in temperature of the probe to the temperature of the gold wire. Both experimental and FEA simulation results indicate that $\eta<0.035$, and that $\eta$ can varies strongly from probe to probe due to geometric variations. These results emphasize the necessity of calibrating each probe individually by scanning over a sample with a known temperature profile. Additionally, because the temperature sensitivity increases with increasing probe tip curvature, a compromise in the probe design has to be made between improving spatial resolution by decreasing the tip curvature, and enhancing temperature sensitivity by increasing the curvature. FEA indicates that temperature sensitivity can also be improved using a narrower metallization design on the sharp end of the tip like the bow-tie shape explored in the simulations.

\section{ACKNOWLEDGMENTS}

The authors thank Dr. Weibin Zhu for help with scanning probe fabrication and Dr. Ben Schrag and the National Science Foundation for their support (NSF Award No. 1010218).

${ }^{1}$ M.-H. Li and Y. B. Gianchandani, Sens. Actuators A 104, 236 (2003).

${ }^{2}$ M.-H. Li, J. J. Wu, and Y. B. Gianchandani, J. Microelectromech. Syst. 10, 3 (2001).

${ }^{3}$ L. E. Ocola, D. Fryer, P. Nealey, J. dePablo, F. Cerrina, and S. Kammer, J. Vac. Sci. Technol. B 14, 3974 (1996).

${ }^{4}$ A. Hammiche, H. M. Pollock, M. Song, and D. J. Hourston, Meas. Sci. Technol. 7, 142 (1996).

${ }^{5}$ K. Luo, Z. Shi, J. Lai, and A. Majumdar, Appl. Phys. Lett. 68, 325 (1996).

${ }^{6}$ J. Lai and A. Majumdar, IEEE Electron Device Lett. 16, 312 (1995).

${ }^{7}$ A. Hammiche, H. M. Pollock, M. Reading, M. Claybourn, P. H. Turner, and K. Jewkes, Appl. Spectrosc. 53(7), 810 (1999).

${ }^{8}$ P. Vettiger, M. Despont, U. Drechsler, U. Durig, W. Haberle, M. I. Lutwyche, H. E. Rothuizen, R. Stutz, R. Widmer, and G. K. Binnig, IBM J. Res. Develop. 44, 323 (2000).

${ }^{9}$ J. Lerchner, D. Caspary, and G. Wolf, Sens. Actuators B 70, 57 (2000).

${ }^{10}$ A. Majumdar, Annu. Rev. Mater. Sci. 29, 505 (1999).

${ }^{11}$ B. A. Nelson and W. P. King, Sens. Actuators A 140, 51 (2007).

${ }^{12}$ H. Fischer, J. Therm. Anal. Calorim. 92(2), 625 (2008).

${ }^{13}$ B. W. Chui, T. D. Stowe, Y. S. Ju, K. E. Goodson, T. W. Kenny, H. J. Mamin, B. D. Terris, R. P. Ried, and D. Rugar, J. Microelectromech. Syst. 7, 69 (1998).

${ }^{14}$ J. Lee and Y. B. Gianchandani, Rev. Sci. Instrum. 75, 1222 (2004).

${ }^{15}$ D. S. Fryer, P. F. Nealey, and J. J. de Pablo, Macromolecules 33, 6439 (2000).

${ }^{16}$ J. Lee and Y. B. Gianchandani, J. Microelectromech. Syst. 14, 44 (2005).

${ }^{17}$ A. Gaitas, S. Gianchandani, and W. Zhu, Rev. Sci. Instrum. 82, 053701 (2011).

${ }^{18}$ A. Gaitas, T. Li, and W. Zhu, Sens. Actuators, A 168, 2 (2011).

${ }^{19}$ A. Gaitas and P. French, Sens. Actuators, A 186, 125 (2012).

${ }^{20}$ W. M. Rohsenow, J. P. Hartnett, and Y. I. Cho, Handbook of Heat Transfer (McGraw-Hill, New York, 1998).

${ }^{21}$ L. Shi and A. Majumdar, J. Heat Transfer 124, 329 (2002). 